\title{
EXISTENCE, UNIQUENESS, AND STABILITY OF OSCILLATIONS IN DIFFERENTIAL EQUATIONS WITH ASYMMETRIC NONLINEARITIES
}

\author{
A. C. LAZER AND P. J. MCKENNA
}

\begin{abstract}
We give conditions for the existence, uniqueness, and asymptotic stability of periodic solutions of a second-order differential equation with piecewise linear restoring and $2 \pi$-periodic forcing where the range of the derivative of the restoring term possibly contains the square of an integer. With suitable restrictions on the restoring and forcing in the undamped case, we give a necessary and sufficient condition.
\end{abstract}

\section{INTRODUCTION}

The main motivation for this paper comes from a numerical study carried out in [5]. Consider the partial differential equation

$$
\partial^{2} U / \partial t^{2}+k \partial U / \partial t+c \partial^{4} U / \partial x^{4}+d U^{+}=h(t, x),
$$

where $U=U(x, t), 0 \leq x \leq L, t \geq 0$ satisfies the boundary conditions

$$
U(0, t)=U(L, t)=\left(\partial^{2} U / \partial x^{2}\right)(0, t)=\left(\partial^{2} U / \partial x^{2}\right)(L, t)=0,
$$

$c>0, k \geq 0, d \geq 0$ are constants, and $U^{+}$denotes the positive part of $U$. If $k=d=0$, then (1.1) and (1.2) describe the transverse vibrations of a beam of length $L$ which is hinged at both ends and where $h(x, t)$ is the external force acting on the beam.

In [5], the boundary value problem (1.1), (1.2) was suggested as a model for an idealized suspension bridge. The term $d U^{+}$takes into account the fact that when the cables suspending the bridge are stretched, there is a restoring force which is assumed to be proportional to the amount of stretching (Hooke's law). But when the beam moves in the opposite direction, then there is no restoring force exerted on it. The term $k \partial U / \partial t$ represents viscuous damping, assumed to be small. The same model with $k=0$ had been considered previously in [10].

Received by the editors March 23, 1988.

1980 Mathematics Subject Classification (1985 Revision). Primary 34C25.

Key words and phrases. Unique periodic solution, continuation argument, implicit function theorem.

The first author was partially supported by the NSF under Grant DMS8519882.

The second author was partially supported by the NSF under Grant DMS8519726. 
The purpose of [5] was to show how such a model could predict the existence of large amplitude, stable oscillations in suspension bridges. Assume that the external force is of the form

$$
h(x, t)=(\sin \pi x / L)(s+f(t)),
$$

where $s>0$ is a constant and $f(t)$ is $T$-periodic in $t, T>0$, with meanvalue zero. If we look for a standing-wave solution of (1.1), (1.2) having the form

$$
U(x, t)=(\sin \pi x / L) u(t)
$$

(this corresponds to a no-nodal motion of the bridge) we note that, because of the positive homogeneity of the nonlinear term, such a solution will exist if and only if $u(t)$ is a solution of the O.D.E.

$$
u^{\prime \prime}(t)+k u^{\prime}(t)+c(\pi / L)^{4} u(t)+d u^{+}(t)=s+f(t) .
$$

Assume $k>0$ and that $f$ is continuous and let $w(t)$ be the unique $T$ periodic solution of the linear differential equation

$$
w^{\prime \prime}+k w^{\prime}+\left[c(\pi / L)^{4}+d\right] w=f(t) .
$$

If $s>0$ and the amplitude of $f(t)$ is small enough to ensure that

$$
u_{0}(t)=s /\left[c(\pi / L)^{4}+d\right]+w(t) \geq 0
$$

for all $t$, then $u_{0}(t)$ is an asymptotically stable $T$-periodic solution of (1.3). The solution $U(x, t)=(\sin \pi x / L) u_{0}(t)$ of $(1.1),(1.2)$ represents small oscillations about the equilibrium solution $\left[c(\pi / L)^{4}+d\right] \sin \pi x / L$ of $(1.1),(1.2)$ when the small oscillatory term $f(t) \equiv 0$.

Using extensions of results of Loud in [13] (Loud considered restoring forces of class $C^{1}$ ) it was shown in [5] that if the form and period of $f$ were suitably restricted, if $k>0$ was sufficiently small, and if the amplitude of $f(t)$ was sufficiently small, then there was also a large-amplitude, asymptotically stable, $T$-periodic solution of (1.3) near a translate of a nonconstant $T$-periodic solution of the autonomous O.D.E.

$$
u^{\prime \prime}+k u^{\prime}+c(\pi / L)^{4} u+d u^{+}=s .
$$

For convenience, let us write

$$
a=c(\pi / L)^{4}, \quad b=d+c(\pi / L)^{4} .
$$

The results of [5] show that if $A>0, \mu>0, \gamma$ is arbitrary,

$$
2 \pi / \sqrt{b}<2 \pi / \mu<\pi / \sqrt{a}+\pi / \sqrt{b}
$$

and the ratio $A / s$ and $k>0$ are sufficiently small, then

$$
u^{\prime \prime}+k u^{\prime}+b u^{+}-a u^{-}=s+A \sin (\mu t+\gamma)
$$


will have exactly two stable $2 \pi / \mu$-periodic solutions, one of which is close to the constant $s / b$ and the other close to a nonconstant $2 \pi / \mu$-periodic solution of

$$
u^{\prime \prime}+b u^{+}-a u^{-}=s .
$$

In [5] a numerical study of the differential equation

$$
u^{\prime \prime}+(.01) u^{\prime}+17 u^{+}-13 u^{-}=10+A \sin \mu t
$$

was made. With $\mu=4$, it appeared that for sufficiently large $A \quad(A \geq .61)$ there was a unique stable periodic solution.

In this paper we give some mathematical justification to the conclusion of the above-mentioned numerical experiment. In Theorem 1 below we show that if $a$ and $b$ are constants satisfying

$$
\frac{(2 m-1)^{2}}{4}<a \leq b<\frac{(2 m+1)^{2}}{4},
$$

where $m \geq 1$ is an integer, $k>0, \gamma$ is arbitrary, and $p(t)$ is a $2 \pi$-periodic function of class $C^{1}$ such that

$$
\int_{0}^{2 \pi} p(t) \cos m t d t=\int_{0}^{2 \pi} p(t) \sin m t d t=0,
$$

then if $A>0$ is sufficiently large, there exists a unique $2 \pi$-periodic solution of

$$
u^{\prime \prime}+k u^{\prime}+b u^{+}-a u^{-}=p(t)+A \sin (m t+\gamma) \text {. }
$$

Moreover, this solution is asymptotically stable.

We emphasize that we have only been able to establish local asymptotic stability. Unless $b-a$ is sufficiently small (so the O.D.E. is close to linear) we feel that it is likely that there exist subharmonic and/or almost periodic solutions. We also emphasize that the damping $k$ does not have to be small.

In Theorem 2 below we consider the undamped differential equation

$$
u^{\prime \prime}+b u^{+}-a u^{-}=A \sin (m t+\gamma),
$$

where $A>0$. Together with Lemma 2.3 below, Theorem 2 shows that if $a$ and $b$ satisfy the restrictions given below, then (1.6) has a periodic solution if and only if

$$
1 / \sqrt{a}+1 / \sqrt{b} \neq 2 / m \text {. }
$$

In fact, if this condition does not hold, then (1.6) has no bounded solutions. This, of course, is well known if $a=b=m^{2}$.

In connection with Theorem 2, we mention the interesting work of Gallouët and Kavian [3, 4]. They consider equations of the form

$$
L u=g(u)+s \theta+h,
$$

where $L$ is a selfadjoint operator with compact resolvent on a real Hilbert space, $g: \mathbf{R} \rightarrow \mathbf{R}$ satisfies

$$
g(\xi) / \xi \rightarrow \begin{cases}a & \text { as } \xi \rightarrow-\infty \\ b & \text { as } \xi \rightarrow \infty\end{cases}
$$


$c \leq a, b \leq d$, where the interval $[c, d]$ contains exactly one simple eigenvalue of $L, \theta$ is an eigenfunction corresponding to this eigenvalue, $s$ is parameter, and $h$ is orthogonal to $\theta$. The condition that the eigenvalue contained in $[c, d]$ is essential in the arguments in [3,4].

In our case $m^{2}$ is a double eigenvalue of the problem

$$
-u^{\prime \prime}=\lambda u, \quad u(0)=u(2 \pi), \quad u^{\prime}(0)=u^{\prime}(2 \pi) .
$$

However, as in [3] and [4] we do make use of the Liapunov-Schmidt reduction method.

We remark that by a simple change of the time scale, we can consider forcing which has period different from $2 \pi$. This will result in different restrictions on $a$ and $b$.

2

In this section $m \geq 1$ will denote a fixed integer and $\gamma_{1}$ and $\gamma_{2}$ fixed numbers such that

$$
\frac{(2 m-1)^{2}}{4}<\gamma_{1}<\gamma_{2}<\frac{(2 m+1)^{2}}{4} \text {. }
$$

If $g$ is a real valued function defined on some interval $I$, then $\chi(g)$ will denote the characteristic function of the set

$$
\{t \in I \mid g(t)>0\} \text {. }
$$

We consider the differential equation

$$
u^{\prime \prime}+k u^{\prime}+b u^{+}-a u^{-}=\cos m t
$$

with $a, b$ and $k$ constants, where $u^{+}\left(u^{-}\right)$denotes the positive (negative) part of $u$. Our main tool in this section is the following

Lemma 2.1. If

$$
\gamma_{1} \leq a \leq b \leq \gamma_{2}
$$

and $u_{0}(t)$ is a $2 \pi$-periodic solution of (2.2), then there exists no nonzero $4 \pi$ periodic solution of

$$
L(y) \equiv y^{\prime \prime}+k y^{\prime}+\left[b \chi\left(u_{0}^{+}\right)+a \chi\left(u_{0}^{-}\right)\right] y=0 .
$$

(By a solution of (2.4) we shall mean a $C^{1}$-function $y$ with $y^{\prime}$ absolutely continuous such that (2.4) holds almost everywhere.)

Proof. Suppose, contrary to the assertion of the lemma, there exists a nonzero $4 \pi$-periodic solution $y_{0}(t)$ of $(2.4)$. If

$$
A(t)=\left[\begin{array}{cc}
0 & 1 \\
-p(t) & -k
\end{array}\right],
$$

where

$$
p(t)=\left[b \chi\left(u_{0}^{+}\right)+a \chi\left(u_{0}^{-}\right)\right](t),
$$


then the column matrix

$$
\bar{y}(t)=\left[\begin{array}{l}
y_{0}(t) \\
y_{0}^{\prime}(t)
\end{array}\right]
$$

is a nontrivial $4 \pi$-periodic solution of the homogeneous linear system $\bar{x}^{\prime}(t)=$ $A(t) \bar{x}(t)$. It is known that the adjoint system $\bar{x}^{\prime}(t)^{T}=-\bar{x}(t)^{T} A(t)$ has a nontrivial $4 \pi$-periodic solution $\bar{z}(t)^{T}=[v(t), w(t)]$. (See for example [6, p. 146].) It follows that $v^{\prime}=p(t) w$ and $w^{\prime}=-v+k w$, and therefore $w$ is a nontrivial $4 \pi$-periodic solution of

$$
L^{*}(y) \equiv y^{\prime \prime}-k y^{\prime}+p(t) y=0
$$

Since

$$
b u_{0}^{+}(t)-a u_{0}^{-}(t)=\left[b \chi\left(u_{0}^{+}\right)+a \chi\left(u_{0}^{-}\right)\right](t) u_{0}(t)
$$

it follows from (2.2) that

$$
L\left(u_{0}\right)=u_{0}^{\prime \prime}+k u_{0}^{\prime}+p(t) u_{0}=\cos m t .
$$

From this it follows that since both $u_{0}$ and $w$ are $4 \pi$-periodic and $L^{*}(w)=0$ we have that

$$
\int_{0}^{4 \pi} w(t) \cos m t d t=\int_{0}^{4 \pi} w(t) L\left(u_{0}\right)(t) d t=\int_{0}^{4 \pi} u_{0}(t) L^{*}(w)(t) d t=0
$$

Since any accumulation point of zeros of $u_{0}$ must also be an accumulation point of zeros of $u_{0}^{\prime}$ as well as an accumulation point of zeros of $u_{0}^{\prime \prime}$, it follows from (2.2) that the only possible accumulation points of zeros of $u_{0}$ are the values of $t$ for which $\cos m t=0$. From (2.2) it follows that $u_{0}$ has a third derivative on the intervals on which $u_{0}(t) \neq 0$ and differentiating (2.2) we obtain

$$
u_{0}^{\prime \prime \prime}+k u_{0}^{\prime \prime}+\left[b \chi\left(u_{0}^{+}\right)+a \chi\left(u_{0}^{-}\right)\right] u_{0}^{\prime}=-m \sin m t .
$$

Since the accumulation points of the zeros of $u_{0}$ are isolated we see that $u_{0}^{\prime \prime \prime}$ is Riemann-integrable and from (2.7) we have that

$$
\begin{aligned}
-m \int_{0}^{4 \pi} w(t) \sin m t d t & =\int_{0}^{4 \pi} w(t) L\left(u_{0}^{\prime}\right)(t) d t \\
& =\int_{0}^{4 \pi} u_{0}^{\prime}(t) L^{*}(w)(t) d t=0 .
\end{aligned}
$$

To conclude the proof we use a variant of a technique which was used for variational type problems in [8] and for a nonvariational type problem in [14]. Representing the $4 \pi$-periodic function $w(t)$ by its uniformly convergent Fourier series

$$
w(t)=c_{0}+\sum_{j=1}^{\infty} c_{j} \cos \frac{j}{2} t+d_{j} \sin \frac{j}{2} t
$$


it follows from (2.6) and (2.8) that $c_{2 m}=d_{2 m}=0$. We can therefore write $w=w_{1}+w_{2}$, where

$$
\begin{aligned}
& w_{1}(t)=c_{0}+\sum_{j=1}^{2 m-1} c_{j} \cos \frac{j}{2} t+d_{j} \sin \frac{j}{2} t, \\
& w_{2}(t)=\sum_{j=2 m+1}^{\infty} c_{j} \cos \frac{j}{2} t+d_{j} \sin \frac{j}{2} t .
\end{aligned}
$$

Let

$$
(f, g)_{0}=\int_{0}^{4 \pi} f(t) g(t) d t
$$

and let $|f|_{0}^{2}=(f, f)_{0}$. From Parseval's identity we have

$$
\begin{aligned}
\left|w_{1}^{\prime}\right|_{0}^{2} & =2 \pi \sum_{j=1}^{2 m-1} \frac{j^{2}}{4}\left(c_{j}^{2}+d_{j}^{2}\right) \\
& \leq \frac{(2 m-1)^{2}}{4}\left[4 \pi c_{0}^{2}+2 \pi \sum_{j=1}^{2 m-1}\left(c_{j}^{2}+d_{j}^{2}\right)\right]=\frac{(2 m-1)^{2}}{4}\left|w_{1}\right|_{0}^{2}
\end{aligned}
$$

and

$$
\begin{aligned}
\left|w_{2}^{\prime}\right|_{0}^{2} & =2 \pi \sum_{2 m+1}^{\infty} \frac{j^{2}}{4}\left(c_{j}^{2}+d_{j}^{2}\right) \\
& \geq 2 \pi \frac{(2 m+1)^{2}}{4} \sum_{j=2 m+1}^{\infty}\left(c_{j}^{2}+d_{j}^{2}\right)=\frac{(2 m+1)^{2}}{4}\left|w_{2}\right|_{0}^{2} .
\end{aligned}
$$

Clearly

$$
\left(w_{1}^{\prime}, w_{2}\right)_{0}=\left(w_{2}^{\prime}, w_{1}\right)_{0}=0
$$

and for $i=1,2$

$$
\left(w_{i}^{\prime}, w_{i}\right)_{0}=\frac{1}{2}\left(w_{i}^{2}(4 \pi)-w_{i}^{2}(0)\right)=0 .
$$

Therefore if we set $z=w_{2}-w_{1}$ we have $\left(z, w^{\prime}\right)_{0}=0$. Since integration by parts gives $-\left(z, w^{\prime \prime}\right)_{0}=\left(z^{\prime}, w^{\prime}\right)_{0}$ we have

$$
\begin{aligned}
0 & =-\left(z, L^{*}(w)\right)_{0} \\
& =\int_{0}^{4 \pi} z^{\prime}(t) w^{\prime}(t)+k \int_{0}^{4 \pi} z(t) w^{\prime}(t) d t-\int_{0}^{4 \pi} p(t) z(t) w(t) d t \\
& =\int_{0}^{4 \pi}\left(w_{2}^{\prime}(t)^{2}-w_{1}^{\prime}(t)^{2}\right) d t-\int_{0}^{4 \pi} p(t)\left(w_{2}(t)^{2}-w_{1}(t)^{2}\right) d t
\end{aligned}
$$

Hence

$$
\int_{0}^{4 \pi}\left(w_{2}^{\prime}(t)^{2}-p(t) w_{2}(t)^{2}\right) d t=\int_{0}^{4 \pi}\left(w_{1}^{\prime}(t)^{2}-p(t) w_{1}(t)^{2}\right) d t
$$


As observed above, the accumulation points of zeros of $u_{0}(t)$ are isolated so from (2.3) and (2.5) it follows that $\gamma_{1} \leq p(t) \leq \gamma_{2}$ almost everywhere on $[0,4 \pi]$. Therefore, from $(2.1),(2.9),(2.10)$ and $(2.11)$ we see that

$$
\begin{aligned}
0 & \leq\left(\frac{(2 m+1)^{2}}{4}-\gamma_{2}\right)\left|w_{2}\right|_{0}^{2} \leq \int_{0}^{4 \pi}\left(w_{2}^{\prime}(t)^{2}-p(t) w_{2}(t)^{2}\right) d t \\
& =\int_{0}^{4 \pi}\left(w_{1}^{\prime}(t)^{2}-p(t) w_{1}(t)^{2}\right) d t \leq\left(\frac{(2 m-1)^{2}}{4}-\gamma_{1}\right)\left|w_{1}\right|_{0}^{2} \leq 0 .
\end{aligned}
$$

Therefore, (2.1) implies that $w_{1}(t) \equiv 0$ and $w_{2}(t) \equiv 0$ so $w(t) \equiv 0$ and we have a contradiction. This contradiction proves the lemma.

Lemma 2.2. Let $f(t, \alpha)$ be continuous for $t \geq 0$ and $0 \leq \alpha \leq \bar{\alpha}$. Let $a(s)$ and $b(s)$ be continuous functions of the parameter $s$ for $0 \leq s \leq 1$ such that $0 \leq a(s) \leq b(s)$. Suppose that for each $\alpha \in[0, \bar{\alpha}]$ the zeros of $f(t, \alpha)$ are isolated on $0 \leq t<\infty$. If $u(t, \xi, \eta, s, \alpha)$ denotes the solution of

$$
u^{\prime \prime}+k u^{\prime}+b(s) u^{+}-a(s) u^{-}=f(t, \alpha), \quad u(0)=\xi, u^{\prime}(0)=\eta,
$$

then the partial derivatives $\frac{\partial u}{\partial \xi}, \frac{\partial u}{\partial \eta}, \frac{\partial u^{\prime}}{\partial \xi}, \frac{\partial u^{\prime}}{\partial \eta}$ exist and are continuous. Moreover, if $u_{0}(t)=u\left(t, \xi_{0}, \eta_{0}, s_{0}, \alpha_{0}\right)$ and

$$
X(t)=\left[\begin{array}{ll}
\frac{\partial u}{\partial \xi}\left(t, \xi_{0}, \eta_{0}, s_{0}, \alpha_{0}\right) & \frac{\partial u}{\partial \eta}\left(t, \xi_{0}, \eta_{0}, s_{0}, \alpha_{0}\right) \\
\frac{\partial u^{\prime}}{\partial \xi}\left(t, \xi_{0}, \eta_{0}, s_{0}, \alpha_{0}\right) & \frac{\partial u^{\prime}}{\partial \eta}\left(t, \xi_{0}, \eta_{0}, s_{0}, \alpha_{0}\right)
\end{array}\right],
$$

then

$$
X^{\prime}(t)=A(t) X(t), \quad X(0)=\left[\begin{array}{ll}
1 & 0 \\
0 & 1
\end{array}\right],
$$

where

$$
A(t)=\left[\begin{array}{cc}
0 & 1 \\
-p(t) & -k
\end{array}\right]
$$

with $p(t)$ given by $(2.5)$.

Although this result seems clear formally, it does not follow from standard theory since the nonlinear function $\xi \rightarrow b(s) \xi^{+}-a(s) \xi^{-}$is not of class $C^{1}$. The proof is given in an appendix at the end of this section.

Lemma 2.3. Assume that the hypotheses of Lemma 2.2 hold and that $f(t, \alpha)$ is $2 \pi$-periodic in $t$. Moreover, assume that for all $s \in[0,1]$

$$
\gamma_{1} \leq a(s) \leq b(s) \leq \gamma_{2},
$$

where $\gamma_{1}$ and $\gamma_{2}$ are as in (2.1). If either

$$
k \neq 0
$$

or

$$
k=0, \quad \frac{1}{\sqrt{a(s)}}+\frac{1}{\sqrt{b(s)}} \neq \frac{2}{m}
$$


for all $s \in[0,1]$, then there exists a number $R>0$ such that if $0 \leq \alpha \leq \bar{\alpha}$, $0 \leq s \leq 1$ and $u(t, \xi, \eta, s, \alpha)$ is $2 \pi$-periodic in $t$, then

$$
\xi^{2}+\eta^{2} \leq R^{2}
$$

Proof. First let us note that (2.1) and (2.3) imply that

$$
\frac{4}{2 m+1}<\frac{2}{\sqrt{\gamma_{2}}} \leq \frac{1}{\sqrt{a(s)}}+\frac{1}{\sqrt{b(s)}}<\frac{2}{\sqrt{\gamma_{1}}}<\frac{4}{2 m-1} .
$$

Assuming that the assertion of the lemma is not true, then either (2.14) or (2.15) holds and there exist sequences $\left\{\xi_{n}\right\}^{\infty},\left\{\eta_{n}\right\}_{1}^{\infty},\left\{s_{n}\right\}_{1}^{\infty}$ and $\left\{\alpha_{n}\right\}_{1}^{\infty}$ such that

$$
\xi_{n}^{2}+\eta_{n}^{2} \rightarrow \infty \text { as } n \rightarrow \infty,
$$

$0 \leq s_{n} \leq 1,0 \leq \alpha_{n} \leq \bar{\alpha}$, and $u\left(t, \xi_{n}, \eta_{n}, s_{n}, \alpha_{n}\right)$ is $2 \pi$-periodic in $t$. By considering suitable subsequences, we may assume that there exist numbers $\xi_{0}$ and $\eta_{0}$ with $\xi_{0}^{2}+\eta_{0}^{2}=1$ and numbers $s_{0} \in[0,1]$ and $\alpha_{0} \in[0, \bar{\alpha}]$ such that

$$
\begin{aligned}
\lim _{n \rightarrow \infty} \frac{\xi_{n}}{\sqrt{\xi_{n}^{2}+\eta_{n}^{2}}} & =\xi_{0}, & & \lim _{n \rightarrow \infty} \frac{\eta_{n}}{\sqrt{\xi_{n}^{2}+\eta_{n}^{2}}}=\eta_{0}, \\
\lim _{n \rightarrow \infty} s_{n} & =s_{0}, & & \lim _{n \rightarrow \infty} \alpha_{n}=\alpha_{0} .
\end{aligned}
$$

If

then, by $(2.12)$

$$
v_{n}(t)=u\left(t, \xi_{n}, \eta_{n}, s_{n}, \alpha_{n}\right) / \sqrt{\xi_{n}^{2}+\eta_{n}^{2}}
$$

$$
v_{n}^{\prime \prime}+k v_{n}^{\prime}+b\left(s_{n}\right) v_{n}^{+}-a\left(s_{n}\right) v_{n}^{-}=f\left(t, \alpha_{n}\right) / \sqrt{\xi_{n}^{2}+\eta_{n}^{2}}
$$

Since the nonlinearity in this differential equation is Lipschitzian, it follows from standard theory (see, for example, [1]) that if $v(t)$ is the function satisfying

$$
v^{\prime \prime}+k v^{\prime}+b\left(s_{0}\right) v^{+}-a\left(s_{0}\right) v^{-}=0, \quad v(0)=\xi_{0}, \quad v^{\prime}(0)=\eta_{0},
$$

then $v_{n}(t) \rightarrow v(t)$ and $v_{n}^{\prime}(t) \rightarrow v^{\prime}(t)$ uniformly on compact subintervals of $(-\infty, \infty)$. Therefore, $v(t+2 \pi) \equiv v(t)$. Since

$$
\begin{aligned}
0 & =\int_{0}^{2 \pi} \frac{d}{d t}\left[v^{\prime}(t)^{2}+\frac{1}{2}\left(b\left(s_{0}\right) v^{+}(t)^{2}+a\left(s_{0}\right) v^{-}(t)^{2}\right] d t\right. \\
& =-k \int_{0}^{2 \pi} v^{\prime}(t)^{2} d t
\end{aligned}
$$

and $v(0)^{2}+v^{\prime}(0)^{2}=1$, it follows that $k=0$. Therefore, (2.15) holds.

On intervals on which $v(t)$ is positive we have $v^{\prime \prime}(t)+b\left(s_{0}\right) v(t)=0$. Therefore, the distance between two consecutive zeros of $v(t)$ which border an interval on which $v(t)$ is positive is $\pi / \sqrt{b\left(s_{0}\right)}$. Similarly, on intervals on which $v(t)$ is negative we have $v^{\prime \prime}(t)+a\left(s_{0}\right) v(t)=0$, so the distance between two 
consecutive zeros of $v(t)$ which border an interval on which $v(t)$ is negative is $\pi / \sqrt{a\left(s_{0}\right)}$. Since

$$
v^{\prime}(t)^{2}+\frac{1}{2}\left[b\left(s_{0}\right) v^{+}(t)^{2}+a\left(s_{0}\right) v^{-}(t)^{2}\right]=\text { constant }
$$

the least period of $v(t)$ is equal to $\pi / \sqrt{a\left(s_{0}\right)}+\pi / \sqrt{b\left(s_{0}\right)}$. But since $2 \pi$ is also a period of $v(t)$, we must have, for some integer $N$,

$$
N\left(\pi / \sqrt{a\left(s_{0}\right)}+\pi / \sqrt{b\left(s_{0}\right)}\right)=2 \pi \text {. }
$$

From (2.17) we see that this implies that $N=m$ which contradicts (2.15). This proves the lemma.

Lemma 2.4. Assume that conditions (2.1) and (2.3) hold. If either (2.14) or

$$
k=0, \quad \frac{1}{\sqrt{a}}+\frac{1}{\sqrt{b}} \neq \frac{2}{m}
$$

hold, then (2.2) has a unique $2 \pi$-periodic solution $\hat{u}(t)$. If $k>0$,

$$
\begin{gathered}
\hat{p}(t)=\left[b \chi\left(\hat{u}^{+}\right)+a \chi\left(\hat{u}^{-}\right)\right](t), \\
B(t)=\left[\begin{array}{cc}
0 & 1 \\
-\hat{p}(t) & -k
\end{array}\right]
\end{gathered}
$$

and $Y(t)$ denotes the $2 \times 2$ matrix such that

$$
Y^{\prime}(t)=B(t) Y(t), \quad Y(0)=I,
$$

then the eigenvalues of $Y(2 \pi)$ have moduli less than 1 .

Proof. If $k \neq 0$ let

$$
a(s)=a, \quad b(s)=b+s(a-b)
$$

for $s \in[0,1]$. If $k=0$ and $1 / \sqrt{a}+1 / \sqrt{b}>2 / m$ let

$$
a(s)=a, \quad b(s)=b+s(a-b)
$$

for $s \in[0,1]$. If $k=0$ and $1 / \sqrt{a}+1 / \sqrt{b}<2 / m$ let

$$
a(s)=a+s(b-a), \quad b(s)=b
$$

for $s \in[0,1]$.

Since (2.3) holds, it is clear that (2.13) holds and either (2.14) holds or (2.15) holds for all $s \in[0,1]$.

To prove the lemma we use a continuation argument similar to one used in [12]. Let $u(t, \xi, \eta, s)$ denote the solution of the initial value problem

$$
\begin{gathered}
u^{\prime \prime}+k u^{\prime}+b(s) u^{+}-a(s) u^{-}=\cos m t, \\
u(0)=\xi, \quad u^{\prime}(0)=\eta .
\end{gathered}
$$

Let

$$
F(\xi, \eta, s)=u(2 \pi, \xi, \eta, s)-\xi, \quad G(\xi, \eta, s)=u^{\prime}(2 \pi, \xi, \eta, s)-\eta
$$


According to Lemma 2.2, $F$ and $G$ are continuous and have continuous partial derivatives with respect to $\xi$ and $\eta$. By standard theory, $u(t, \xi, \eta, s)$ is a $2 \pi$ periodic solution of (2.20) if and only if

$$
F(\xi, \eta, s)=G(\xi, \eta, s)=0 .
$$

Suppose that $F\left(\xi_{0}, \eta_{0}, s_{0}\right)=G\left(\xi_{0}, \eta_{0}, s_{0}\right)=0$ where $s_{0} \in[0,1]$. Let $u_{0}(t)=$ $u\left(t, \xi_{0}, \eta_{0}, s_{0}\right)$. According to Lemma 2.2, we have

$$
\left[\begin{array}{ll}
\partial F / \partial \xi & \partial F / \partial \eta \\
\partial G / \partial \xi & \partial G / \partial \eta
\end{array}\right]\left(\xi_{0}, \eta_{0}, s_{0}\right)=X(2 \pi)-I,
$$

where $X(t)$ is as in the statement of Lemma 2.2 .

We assert that

$$
\operatorname{det}(X(2 \pi)-I) \neq 0 .
$$

Assuming the contrary, there exists a column vector $\bar{c} \neq \overline{0}$ such that $X(2 \pi) \bar{c}=$ $\bar{c}$. It follows that $\bar{y}(t) \equiv X(t) \bar{c}$ is $2 \pi$-periodic, not identically zero, and satisfies $\bar{y}^{\prime}(t)=A(t) \bar{y}(t)$. From this and the form of $A(t)$ we see that

$$
\bar{y}(t)=\left[\begin{array}{c}
y(t) \\
y^{\prime}(t)
\end{array}\right],
$$

where $y(t)$ is nontrivial $2 \pi$-periodic solution of (2.4). But $\gamma_{1} \leq a\left(s_{0}\right) \leq b\left(s_{0}\right) \leq$ $\gamma_{2}$ so according to Lemma $2.1,(2.4)$ has no nontrivial $4 \pi$-periodic solutions. This contradiction proves (2.23).

It follows from (2.22), (2.23) and the inverse function theorem that there exists an open disk $D$ in the $\xi, \eta$-plane centered at $\left(\xi_{0}, \eta_{0}\right)$ such that $\left(F\left(\xi, \eta, s_{0}\right)\right.$, $\left.G\left(\xi, \eta, s_{0}\right)\right) \neq(0,0)$ for $(\xi, \eta) \in D$ and $(\xi, \eta) \neq\left(\xi_{0}, \eta_{0}\right)$. This shows that for each $s \in[0,1]$ the set of points $(\xi, \eta)$ for which $(2.21)$ holds has no limit point. Since according to Lemma 2.3, there exists a number $R>0$ such that $s \in[0,1]$, and (2.21) imply that $\xi^{2}+\eta^{2} \leq R^{2}$, it follows that for each $s \in[0,1]$, (2.21) has only a finite number of solutions.

For each integer $p \geq 0$, let $\Sigma_{p}$ denote the set of numbers $s \in[0,1]$ such that there are exactly $p$ points satisfying (2.21). If $s_{0} \in \Sigma_{p}$ and $\left(\xi_{j}, \eta_{j}\right), j=$ $1, \ldots, p$, denote solutions of $F\left(\xi, \eta, s_{0}\right)=G\left(\xi, \eta, s_{0}\right)=0$ then, since the argument given above implies that the Jacobian of the map $(\xi, \eta) \rightarrow\left(F\left(\xi, \eta, s_{0}\right)\right.$, $\left.G\left(\xi, \eta, s_{0}\right)\right)$ is nonzero at $\left(\xi_{j}, \eta_{j}\right), 1 \leq j \leq p$, a version of the implicit function theorem $[2,16]$ implies the existence of open disjoint disks $D_{j}, j=1, \ldots, p$, and a number $\delta>0$ such that $D_{j}$ is centered at $\left(\xi_{j}, \eta_{j}\right)$ and for $\left|s-s_{0}\right|<\delta$, $s \in[0,1]$, there exists a unique point $\left(\hat{\xi}_{j}(s), \hat{\eta}_{j}(s)\right) \in D_{j}$, varying continuously with $s$, such that

$$
F\left(\hat{\xi}_{j}(s), \hat{\eta},(s), s\right)=G\left(\hat{\xi}_{j}(s), \hat{\eta}_{j}(s), s\right)=0
$$

and $\left(\hat{\xi}_{j}\left(s_{0}\right), \hat{\eta}_{j}\left(s_{0}\right)\right)=\left(\xi_{j}, \eta_{j}\right)$. Since $\left(F\left(\xi, \eta, s_{0}\right), G\left(\xi, \eta, s_{0}\right)\right) \neq(0,0)$ for $(\xi, \eta) \in\left\{(\xi, \eta) \mid \xi^{2}+\eta^{2} \leq R^{2},(\xi, \eta) \notin D_{j}, j=1, \ldots, p\right\} \equiv K$ and $K$ is 
compact, by choosing $\delta>0$ smaller, if necessary, it may be assumed that (2.21) has no solution $(\xi, \eta) \in K$ for $\left|s-s_{0}\right|<\delta$.

It follows that if $s \in[0,1]$ and $\left|s-s_{0}\right|<\delta$, then (2.21) has exactly $p$ solutions $(\xi, \eta)$. Hence $\Sigma_{p}$ is open in $[0,1]$.

Since $\Sigma_{p}$ is disjoint from $\Sigma_{q}$ for $p \neq q$ and since the interval $[0,1]$ is connected, it follows that there exists an integer $p \geq 0$ such that $\Sigma_{q}$ is empty for $q \neq p$ and $[0,1]=\Sigma_{p}$.

To determine $p$ we determine the number of solutions of $(2.20)$ when $s=1$. In case $k \neq 0$, then, by (2.19)(i), when $s=1$ (2.20) becomes

$$
u^{\prime \prime}+k u^{\prime}+a u=\cos m t \text {. }
$$

Clearly this has a unique $2 \pi$-periodic solution so when $k \neq 0$ we have $p=1$. In case $k=0$ and $1 / \sqrt{a}+1 / \sqrt{b}>2 / m$, we must have $a<m^{2}$ and, according to (2.19)(ii), when $s=1(2.20)$ becomes

$$
u^{\prime \prime}+a u=\cos m t \text {. }
$$

Since $(2 m-1)^{2} / 4<\gamma_{1} \leq a<m^{2}$, this has a unique $2 \pi$-periodic solution. Finally, if $1 / \sqrt{a}+1 / \sqrt{b}<2 / m$, we must have $b>m^{2}$ and, according to (2.19)(iii), when $s=1$ (2.20) becomes

$$
u^{\prime \prime}+b u=\cos m t
$$

Since $m^{2}<b \leq \gamma_{2}<(2 m+1)^{2} / 4$, this has a unique $2 \pi$-periodic solution. Therefore, both (2.14) and (2.18) imply that $[0,1]=\Sigma_{1}$.

When $s=0,(2.20)$ becomes (2.2). This proves that (2.2) has a unique $2 \pi$-periodic solution $\hat{u}(t)$.

Since $[0,1]=\Sigma_{1}$ it follows that for each fixed $s \in[0,1]$ there exists a unique point $(\hat{\xi}(s), \hat{\eta}(s))$ such that

$$
(F(\hat{\xi}(s), \hat{\eta}(s), s), G(\hat{\xi}(s), \hat{\eta}(s), s))=(0,0) .
$$

The implicit function theorem as used above implies that $\hat{\xi}(s)$ and $\hat{\eta}(s)$ depend continuously on $s$. Therefore, according to Lemma 2.2, if

$$
Z(t, s) \equiv\left[\begin{array}{ll}
\frac{\partial u}{\partial \xi}(t, \hat{\xi}(s), \hat{\eta}(s), s) & \frac{\partial u}{\partial \eta}(t, \hat{\xi}(s), \hat{\eta}(s), s) \\
\frac{\partial u^{\prime}}{\partial \xi}(t, \hat{\xi}(s), \hat{\eta}(s), s) & \frac{\partial u^{\prime}}{\partial \eta}(t, \hat{\xi}(s), \hat{\eta}(s), s)
\end{array}\right],
$$

then the elements of $Z(t, s)$ are continuous in $t$ and $s$, and if

$$
C(t, s)=\left[\begin{array}{cc}
0 & 1 \\
-p(t, s) & -k
\end{array}\right]
$$

where

$$
p(\cdot, s)=b(s) \chi\left(u^{+}(\cdot, \hat{\xi}(s), \hat{\eta}(s), s)\right)+a(s) \chi\left(u^{-}(\cdot, \hat{\xi}(s), \hat{\eta}(s), s)\right),
$$

then

$$
Z^{\prime}(t, s)=C(t, s) Z(t, s), \quad Z(0, s)=I .
$$


In the remainder of the proof we assume $k>0$. Either

$$
C(t, 1)=\left[\begin{array}{cc}
0 & 1 \\
-a & -k
\end{array}\right] \text { or } C(t, 1)=\left[\begin{array}{cc}
0 & 1 \\
-b & -k
\end{array}\right] \text {. }
$$

In either case if $\lambda_{1}$ and $\lambda_{2}$ are the eigenvalues of the constant matrix $C(t, 1)$, then both $\lambda_{1}$ and $\lambda_{2}$ have negative real parts. Since the eigenvalues of $Z(2 \pi, 1)$ are $e^{\lambda_{2} 2 \pi}$ and $e^{\lambda_{2} 2 \pi}$, the moduli of both eigenvalues of $Z(2 \pi, 1)$ are less than 1 . By elementary considerations (for example, by Rouché's theorem) for $s \in[0,1]$ and $|s-1|$ small, both eigenvalues of $Z(2 \pi, s)$ have moduli less than 1 . If the moduli of both eigenvalues of $Z(2 \pi, s)$ are not less than 1 for all $s \in[0,1]$ then by Rouche's theorem and the continuity of the elements of $Z(2 \pi, s)$, there exists a number $s^{*}$ with $0 \leq s^{*}<1$ such that for $s^{*}<s \leq 1$, both eigenvalues of $Z(2 \pi, s)$ have moduli less than 1 and the eigenvalues of $Z\left(2 \pi, s^{*}\right)$ may be denoted by $\sigma_{1}$ and $\sigma_{2}$ such that $\left|\sigma_{1}\right|=1$ and $\left|\sigma_{2}\right| \leq 1$.

Since the trace of $C(t, s)$ is identically equal to $-k<0$, it follows from Liouville's formula that

$$
\sigma_{1} \sigma_{2}=\operatorname{det} Z\left(2 \pi, s^{*}\right)=e^{-2 \pi k}<1 .
$$

This shows that $\sigma_{1}$ and $\sigma_{2}$ cannot be complex conjugates since this would imply $\left|\sigma_{2}\right|=\left|\sigma_{1}\right|=1$, contradicting the previous equation. Therefore $\sigma_{1}=1$ or $\sigma_{1}=-1$. If $\sigma_{1}=1$ then there exists a column vector $\bar{c} \neq 0$ such that $Z\left(2 \pi, s^{*}\right) \bar{c}=\bar{c}$. As in an argument given before, if $\bar{y}(t)=Z\left(t, s^{*}\right) \bar{c}$ then

$$
\bar{y}(t)=\left[\begin{array}{c}
y(t) \\
y^{\prime}(t)
\end{array}\right],
$$

where $y(t)$ is a nontrivial $2 \pi$-periodic solution of

$$
y^{\prime \prime}(t)+k y^{\prime}(t)+p\left(t, s^{*}\right) y(t)=0 .
$$

But since $(2.13)$ holds and $\hat{u}\left(t, \hat{\xi}\left(s^{*}\right), \hat{\eta}\left(s^{*}\right), s^{*}\right)$ is a $2 \pi$-periodic solution of (2.20), it follows from Lemma 2.1 and the definition of $p\left(t, s^{*}\right)$ that (2.27) can have no nontrivial $4 \pi$-periodic solution and therefore it cannot have a nontrivial $2 \pi$-periodic solution.

We are therefore left only with the possibility that $\sigma_{1}=-1$. This implies the existence of a column vector $\bar{c} \neq 0$ such that $Z\left(2 \pi, s^{*}\right) \bar{c}=-\bar{c}$. It follows that if $\bar{y}(t)=Z\left(t, s^{*}\right) \bar{c}$, then (2.26) holds, where $y(t)$ is a solution of (2.27) such that

$$
y(2 \pi)=-y(0), \quad y^{\prime}(2 \pi)=-y^{\prime}(0) .
$$

Since $y(t+2 \pi)$ and $-y(t)$ are both solutions of (2.27) it follows by these two equations and the uniqueness theorem that

$$
y(t+2 \pi) \equiv-y(t) .
$$

Therefore $y(t)$ is a nontrivial $4 \pi$-periodic solution of (2.27) which contradicts Lemma 2.1. This contradiction shows that the eigenvalues of $Z(2 \pi, s)$ must have moduli less than 1 for $0 \leq s \leq 1$. Since $Z(2 \pi, 0)=Y(t)$ this proves the lemma.

We now prove our main result: 
Theorem 1. Assume that (2.1) and (2.3) hold. Let $h(t)$ be a $2 \pi$-periodic function of class $C^{1}$ such that

$$
\int_{0}^{2 \pi} h(t) \cos m t d t=\int_{0}^{2 \pi} h(t) \sin m t d t=0 .
$$

Let $k>0$ and let $\gamma$ be an arbitrary constant. If $A>0$ is sufficiently large then there exists a unique $2 \pi$-periodic solution of

$$
u^{\prime \prime}+k u^{\prime}+b u^{+}-a u^{-}=h(t)+A \sin (m t+\gamma) .
$$

Moreover, this solution is locally asymptotically stable.

Proof. Since $u(t)$ is a $2 \pi$-periodic solution of equality (2.29) if and only if $u(t+\pi / 2 m-\gamma / m)$ is a $2 \pi$-periodic solution of

$$
u^{\prime \prime}+k u^{\prime}+b u^{+}-a u^{-}=\bar{h}(t)+A \cos m t,
$$

where $\bar{h}(t)=h(t+\pi / 2 m-\gamma / m)$, it is sufficient to consider this latter equation. Since $u(t)$ is a solution of (2.30) if and only if $u(t) / A$ is a solution of

$$
u^{\prime \prime}+k u^{\prime}+b u^{+}-a u^{-}=\alpha \bar{h}(t)+\cos m t,
$$

where $\alpha=1 / A$, to prove the theorem it is sufficient to prove that for $|\alpha|$ sufficiently small, $(2.31)$ has a unique $2 \pi$-periodic solution and that this solution is locally asymptotically stable.

To this end let $\tilde{u}(t, \xi, \eta, \alpha)$ denote the solution of (2.31) such that

$$
\tilde{u}(0, \xi, \eta, \alpha)=\xi, \quad \tilde{u}^{\prime}(0, \xi, \eta, \alpha)=\eta .
$$

Since the zeros of $\cos m t$ are isolated and $\bar{h}(t)$ is a $2 \pi$-periodic function of class $C^{1}$ there exists a number $\alpha_{0}$ such that the zeros of $f(t, \alpha) \equiv \cos m t+\alpha \bar{h}(t)$ are isolated if $|\alpha| \leq \alpha_{0}$. Hence, according to Lemma (2.2) both $\tilde{u}(t, \xi, \eta, \alpha)$ and $\tilde{u}^{\prime}(t, \xi, \eta, \alpha)$ have continuous partial derivatives with respect to $\xi$ and $\eta$ for $|\alpha| \leq \alpha_{0}$.

By standard theory, $u(t, \xi, \eta, \alpha)$ is a $2 \pi$-periodic solution of (2.31) if and only if

$$
\tilde{u}(2 \pi, \xi, \eta, \alpha)=\xi, \quad \tilde{u}^{\prime}(2 \pi, \xi, \eta, \alpha)=\eta .
$$

By Lemma 2.3 there exists a number $R>0$ such that (2.32) implies $\xi^{2}+\eta^{2} \leq$ $R^{2}$. By Lemma 2.4 there exists a unique point $\left(\xi_{0}, \eta_{0}\right)$ such that

$$
\tilde{u}\left(2 \pi, \xi_{0}, \eta_{0}, 0\right)=\xi_{0}, \quad \tilde{u}^{\prime}\left(2 \pi, \xi_{0}, \eta_{0}, 0\right)=\eta_{0} .
$$

Moreover, by the same lemma the eigenvalues of the Jacobian matrix of the mapping

$$
\xi \rightarrow \tilde{u}(2 \pi, \xi, \eta, 0), \quad \eta \rightarrow \tilde{u}(2 \pi, \xi, \eta, 0)
$$

have modulus less than 1 . Therefore the mapping

$$
\xi \rightarrow \tilde{u}(2 \pi, \xi, \eta, \alpha)-\xi, \quad \eta \rightarrow \tilde{u}(2 \pi, \xi, \eta, \alpha)-\eta
$$

has nonzero Jacobian at $(\xi, \eta, \alpha)=\left(\xi_{0}, \eta_{0}, 0\right)$. 
The same version of the implicit function theorem and the same type of argument as used in Lemma 2.4 implies the existence of a number $\alpha_{1}>0$ such that for $|\alpha| \leq \alpha_{1}$ there exists a unique pair of functions $(\bar{\xi}(\alpha), \tilde{\eta}(\alpha))$ which satisfy (2.32). Moreover, $(\hat{\xi}(\alpha), \tilde{\eta}(\alpha))$ depends continuously on $\alpha$ and $\tilde{\xi}(0)=\xi_{0}$ and $\tilde{\eta}(0)=\eta_{0}$.

According to Lemmas 2.2 and 2.4 the eigenvalues of

$$
\left[\begin{array}{ll}
\frac{\partial \tilde{u}}{\partial \xi}\left(2 \pi, \xi_{0}, \eta_{0}, 0\right) & \frac{\partial \tilde{u}}{\partial \eta}\left(2 \pi, \xi_{0}, \eta_{0}, 0\right) \\
\frac{\partial \tilde{u}^{\prime}}{\partial \xi}\left(2 \pi, \xi_{0}, \eta_{0}, 0\right) & \frac{\partial \tilde{u}^{\prime}}{\partial \eta}\left(2 \pi, \xi_{0}, \eta_{0}, 0\right)
\end{array}\right]
$$

have moduli less than 1. By Rouché's theorem, there exists a number $\alpha_{2}>0$ such that if $|\alpha|<\alpha_{2}$, the moduli of the eigenvalues of

$$
\left[\begin{array}{ll}
\frac{\partial \tilde{u}}{\partial \xi}(2 \pi, \tilde{\xi}(\alpha), \tilde{\eta}(\alpha), \alpha) & \frac{\partial \tilde{u}}{\partial \eta}(2 \pi, \tilde{\xi}(\alpha), \tilde{\eta}(\alpha), \alpha) \\
\frac{\partial \tilde{u}^{\prime}}{\partial \xi}(2 \pi, \tilde{\xi}(\alpha), \tilde{\eta}(\alpha), \alpha) & \frac{\partial \tilde{u}^{\prime}}{\partial \eta}(2 \pi, \tilde{\xi}(\alpha), \tilde{\eta}(\alpha), \alpha)
\end{array}\right]
$$

are less than 1. But this means that the unique fixed point $(\tilde{\xi}(\alpha), \tilde{\eta}(\alpha))$ of

$$
(\xi, \eta) \rightarrow\left(\tilde{u}(2 \pi, \xi, \eta, \alpha), \tilde{u}^{\prime}(2 \pi, \xi, \eta, \alpha)\right)
$$

is attracting (see [7, p. 280]). Since this implies that the $2 \pi$-periodic solution $\tilde{u}(t, \tilde{\xi}(\alpha), \tilde{\eta}(\alpha), \alpha)$ is asymptotically stable, the theorem is proved.

We conclude by giving a converse to Lemma 2.4 in the undamped case $k=0$.

Theorem 2. If

$$
(m-1)^{2}<a \leq b<(m+1)^{2}
$$

and

$$
1 / \sqrt{a}+1 / \sqrt{b}=2 / m
$$

then for any $A>0$ and $\gamma$ the differential equation

$$
u^{\prime \prime}+b u^{+}-a u^{-}=A \sin (m t+\gamma)
$$

has no solution bounded on the interval $0 \leq t<\infty$.

Proof. If (2.35) had a solution bounded on $[0, \infty)$, then the second derivative and hence the first derivative would be bounded on $[0, \infty)$. Since the differential equation is $2 \pi$-periodic in $t$ and since the nonlinearity is Lipschitzian and satisfies a linear growth condition, it would follow from a rather deep result due to Massera [15] that (2.35) would have a $2 \pi$-periodic solution. Therefore, to prove the theorem, it is sufficient to show that if (2.33) and (2.34) hold then (2.35) has no $2 \pi$-periodic solution. Replacing $u$ by $u / A$ and $t$ by $t+\pi / 2 m-\gamma / m$ it is sufficient to show that if (2.33) and (2.34) hold, then

$$
u^{\prime \prime}+b u^{+}-a u^{-}=\cos m t
$$

has no $2 \pi$-periodic solution. 
To prove the last assertion we use some ideas due to the authors [11] and Gallouët and Kavian [3, 4]. Let $H$ be the Hilbert space consisting of realvalued $2 \pi$-periodic functions whose restrictions to the interval $[-\pi, \pi]$ belong to $L^{2}[-\pi, \pi]$ and with the $L^{2}[-\pi, \pi]$ inner-product. Let

$$
V=\operatorname{span}\{\cos m t, \sin m t\}
$$

and let $W=V^{\perp}$ (in $H$ ). Let $P: H \rightarrow V$ denote orthogonal projection.

If $L: D(L) \rightarrow H$ is defined by $L u=-u^{\prime \prime}$, then (2.36) is equivalent to the two equations

$$
L w=(I-P)\left[b(v+w)^{+}-a(v+w)^{-}\right]
$$

and

$$
L v=P\left(b(v+w)^{+}-a(v+w)^{-}\right]-\cos m t,
$$

where $w=(I-P) u$ and $v=P u$. Since the spectrum of $L$ restricted to $W$ consists of the numbers $k^{2}$ where $k=0,1,2, \ldots, k \neq m$, it follows from (2.33) and an obvious modification of Proposition 2.1 of [9] that given $v \in V$ there exists a unique $\bar{w}(v) \in W$ such that $\bar{w}(v)$ is a solution of (2.36)(i).

Suppose, contrary to our claim, there exists $u_{0}$ such that $u_{0}$ is a $2 \pi$-periodic solution of (2.36). We have

$$
\begin{aligned}
0 & =\int_{-\pi}^{\pi} \frac{d}{d t}\left[u_{0}^{\prime}(t)^{2}+b u_{0}^{+}(t)^{2}+a u_{0}^{-}(t)^{2}\right] d t \\
& =\int_{-\pi}^{\pi} 2 u_{0}^{\prime}(t)\left[u_{0}^{\prime \prime}(t)+b u_{0}^{+}(t)-a u_{0}^{-}(t)\right] d t \\
& =\int_{-\pi}^{\pi} 2 u_{0}^{\prime}(t) \cos m t d t=2 m \int_{-\pi}^{\pi} u_{0}(t) \sin m t d t .
\end{aligned}
$$

Therefore if $v_{0}=P u_{0}$, then $v_{0}(t)=c \cos t$ for some constant $c$. We must have $c \neq 0$ for otherwise $v_{0}=0$ and, since $w \equiv 0$ satisfies (2.36)(i) when $v=0$, we would have $u_{0}=v_{0}+\bar{w}\left(v_{0}\right)=0$, contradicting the fact that $u_{0}$ is a solution of (2.36).

Since (2.34) holds, the proof of Lemma 2.3 shows that all solutions of

$$
u^{\prime \prime}+b u^{+}-a u^{-}=0
$$

are periodic with least period $2 \pi / m$ and hence $2 \pi$-periodic. If $u_{1}$ is the solution with $u(0)=1$ and $u^{\prime}(0)=0$, then since both $u_{1}(t)$ and $u_{1}(-t)$ are solutions of (2.37) which are equal and have the same derivative at $t=0$, $u_{1}(t)$ must be even. Therefore $\left(P u_{1}\right)(t)=c_{1} \cos t$ for some constant. We must have $c_{1} \neq 0$. Otherwise, setting $w_{1}=(I-P) u_{1}$ we would have

$$
L w_{1}=(I-P)\left[b w_{1}^{+}-a w_{1}^{-}\right] .
$$

But since $w=0$ is a solution of (2.36)(i) when $v=0$, and for each $v \in V$ there is a unique $w \in W$ satisfying (2.36), we must have $w_{1}=0$. Thus $u_{1}=v_{1}+w_{1} \equiv 0$ which is a contradiction since $u_{1}(0)=1$. Thus $c_{1} \neq 0$. 
If $\operatorname{sgn} c_{1}=\operatorname{sgn} c$ then we set $u_{2}(t)=\frac{c}{c_{1}} u_{1}(t)$ and observe that $u_{2}$ is a solution of (2.37) with $P u_{2}=v_{0}$. If $\operatorname{sgn} c_{1} \neq \operatorname{sgn} c$, then $\bar{u}_{1}(t)=u_{1}(t-\pi)$ is a solution of $(2.37)$ with $\left(P \bar{u}_{1}\right)(t)=-\left(P u_{1}\right)(t)=-c_{1} \cos t$. In this case we set $u_{2}(t)=-\frac{c}{c_{1}} u_{1}(t-\pi)$ and observe that $u_{2}$ is a solution of (2.37) with $P u_{2}=v_{0}$.

If $w_{0}=(I-P) u_{0}$ and $w_{2}=(I-P) u_{2}$, then both $w_{0}$ and $w_{2}$ are solutions of $(2.36)(\mathrm{i})$ when $v=v_{0}$. Since $\bar{w}\left(v_{0}\right)$ is the unique solution of $(2.36)(\mathrm{i})$, this implies that $w_{0}=w_{2}$. Hence, $u_{0}=u_{2}$, so $u_{0}$ is a solution of both (2.36) and (2.37), which is absurd. By earlier remarks, this contradiction proves the theorem.

\section{APPENDIX}

Proof of Lemma 2.2. Let $\gamma, s$ and $\alpha$ be fixed and let $\left\{\xi_{n}\right\}_{1}^{\infty}$ be a sequence of numbers such that $\xi_{n} \rightarrow \xi_{0}$ as $n \rightarrow \infty$ and $\xi_{n} \neq \xi_{0}$ for all $n \geq 1$. Let $g(s, \xi)=b(s) \xi^{+}-a(s) \xi^{-}$. Since $0<a(s) \leq b(s), g$ is Lipschitzian in $\xi$ with Lipschitz constant $b(s)$. Let

$$
u_{n}(t)=u\left(t, \xi_{n}, \eta, s, \alpha\right), \quad u_{0}(t)=u\left(t, \xi_{0}, \eta, s, \alpha\right)
$$

and let

for $n \geq 1$.

$$
\psi_{n}(t)=\left(u_{n}(t)-u_{0}(t)\right) /\left(\xi_{n}-\xi_{0}\right)
$$

Since $u_{n}^{\prime \prime}+k u_{n}+g\left(s, u_{n}\right)=f(t, \alpha)$ for $n \geq 0$, we have

$$
\left|\psi_{n}^{\prime \prime}(t)\right| \leq k\left|\psi_{n}^{\prime}(t)\right|+b(s)\left|\psi_{n}(t)\right|
$$

for $n \geq 1$. Moreover, we have $\psi_{n}(0)=1, \psi_{n}^{\prime}(0)=0$ for $n \geq 1$ so for $t \geq 0$

$$
\psi_{n}(t)=1+\int_{0}^{t} \psi_{n}^{\prime}(s) d s, \quad \psi_{n}^{\prime}(t)=\int_{0}^{t} \psi_{n}^{\prime \prime}(s) d s .
$$

Therefore, if $t \geq 0$ and $M=1+k+b(s)$, then from (2.38) we obtain

$$
\left|\psi_{n}(t)\right|+\left|\psi_{n}^{\prime}(t)\right| \leq 1+M \int_{0}^{t}\left(\left|\psi_{n}(s)\right|+\left|\psi_{n}^{\prime}(s)\right|\right) d s .
$$

From Gronwall's lemma, it follows that for $t \geq 0$ and $n \geq 1$ we have

$$
\left|\psi_{n}(t)\right|+\left|\psi_{n}^{\prime}(t)\right| \leq \exp M t .
$$

Let $\bar{t}>0$. From (2.38) and (2.39) it follows that the sequences $\left\{\psi_{n}\right\}_{1}^{\infty}$ and $\left\{\psi_{n}^{\prime}\right\}_{1}^{\infty}$ are equicontinuous and uniformly bounded on $[0, \bar{t}]$, so by Ascoli's lemma there exists a subsequence $\left\{\psi_{n_{j}}(t)\right\}_{1}^{\infty}$ of $\left\{\psi_{n}(t)\right\}_{1}^{\infty}$ and a $C^{1}$-function $z$ such that $\psi_{n_{j}}(t) \rightarrow z(t)$ and $\psi_{n_{j}}^{\prime}(t) \rightarrow z^{\prime}(t)$ as $n_{j} \rightarrow \infty$ uniformly with respect to $t \in[0, \bar{t}]$.

Since

$$
u_{0}^{\prime \prime}+k u_{0}^{\prime}+b(s) u_{0}^{+}-a(s) u_{0}^{-}=f(t, \alpha)
$$

and, by Rolle's theorem, any accumulation point of zeros of $u_{0}$ must be a zero of both $u_{0}^{\prime}$ and $u_{0}^{\prime \prime}$, it follows that any accumulation point of zeros of $u_{0}$ must 
be a zero of $f(\cdot, \alpha)$. Since, by assumption, the zeros of $f(\cdot, \alpha)$ are isolated it follows that $u_{0}(t) \neq 0$ almost everywhere on $[0, \bar{t}]$.

We have $\psi_{n_{j}}^{\prime \prime}+k \psi_{n_{j}}^{\prime}+h_{j}(t)=0$, where

$$
h_{j}(t)=\left(g\left(s, u_{n_{j}}(t)\right)-g\left(s, u_{0}(t)\right)\right) /\left(\xi_{n_{j}}-\xi_{0}\right) .
$$

By standard theory [1], $u_{n_{j}}(t) \rightarrow u_{0}(t)$ as $j \rightarrow \infty$ uniformly on $[0, \bar{t}]$. If $t \in[0, \bar{t}]$ and $u_{0}(t)>0$, then $u_{n_{j}}(t)>0$ for sufficiently large $j$ so $h_{j}(t)=$ $b(s) \psi_{n_{j}}(t)$ for sufficiently large $j$. If $t \in[0, \bar{t}]$ and $u_{0}(t)<0$ then $u_{n_{j}}\left(t_{0}\right)<0$ for sufficiently large $j$ so $h_{j}(t)=a(s) \psi_{n_{j}}(t)$. It follows that for almost all $t$

$$
h_{j}(t) \rightarrow\left[b(s) \chi\left(u_{0}^{+}\right)+a(s) \chi\left(u_{0}^{-}\right)\right] z(t) \equiv p(t) z(t)
$$

as $j \rightarrow \infty$ on $[0, \bar{t}]$. Moreover, since $\left|h_{j}(t)\right| \leq b(s)\left|\psi_{n_{j}}(t)\right|$ the sequence $\left\{h_{j}(t)\right\}$ is bounded on $[0, \bar{t}]$.

Letting $j \rightarrow \infty$ in

$$
\psi_{n_{j}}(t)=1+\int_{0}^{t} \psi_{n_{j}}^{\prime}(s) d s, \quad \psi_{n_{j}}^{\prime}(t)=-\int_{0}^{t}\left(k \psi_{n_{j}}^{\prime}(s)+h_{j}(s)\right) d s
$$

we obtain

$$
z(t)=1+\int_{0}^{t} z^{\prime}(s) d s, \quad z^{\prime}(t)=-\int_{0}^{t}\left(k z^{\prime}(s)+p(s) z(s)\right) d s
$$

so $z^{\prime}(t)$ is absolutely continuous and

$$
z^{\prime \prime}+k z^{\prime}+p(t) z(t)=0, \quad z(0)=1, \quad z^{\prime}(0)=0
$$

almost everywhere on $[0, \bar{t}]$. Since this determines $z(t)$ uniquely, the original sequences $\left\{\psi_{n}(t)\right\}_{1}^{\infty}$ and $\left\{\psi_{n}^{\prime}(t)\right\}_{1}^{\infty}$ must themselves converge uniformly to $z(t)$ and $z^{\prime}(t)$ respectively and we have established the existence of $\partial u / \partial \xi$ and $\partial u^{\prime} / \partial \xi$.

In an entirely similar way one shows that

$$
\partial u / \partial \eta=w, \quad \partial u^{\prime} / \partial \eta=w^{\prime},
$$

where $w^{\prime \prime}+k w^{\prime}+p(t) w=0, w(0)=0$ and $w^{\prime}(0)=1$.

To establish continuity of $\partial u / \partial \xi, \partial u^{\prime} / \partial \xi, \partial u / \partial \eta$ and $\partial u^{\prime} / \partial \eta$, it is sufficient to show that if $\left\{\xi_{n}\right\}_{1}^{\infty},\left\{\eta_{n}\right\}_{1}^{\infty},\left\{s_{n}\right\}_{1}^{\infty}$ and $\left\{\alpha_{n}\right\}_{1}^{\infty}$ are sequences such that

$$
\left(\xi_{n}, \eta_{n}, s_{n}, \alpha_{n}\right) \rightarrow\left(\xi_{0}, \eta_{0}, s_{0}, \alpha_{0}\right)
$$

as $n \rightarrow \infty$, if $u_{n}(t)=u\left(t, \xi_{n}, \eta_{n}, s_{n}, \alpha_{n}\right)$ for $n \geq 0$, if

$$
p_{n}(t)=\left[b\left(s_{n}\right) \chi\left(u_{n}^{+}\right)+a\left(s_{n}\right) \chi\left(u_{n}^{-}\right)\right](t)
$$

for $n \geq 0$, and if

$$
\begin{array}{ll}
y_{n}^{\prime \prime}(t)+k y_{n}^{\prime}(t)+p_{n}(t) y_{n}(t)=0, & n \geq 0, \\
y_{n}(0)=y_{0}(0), \quad y_{n}^{\prime}(0)=y_{0}^{\prime}(0), & n \geq 1,
\end{array}
$$


then for any $\bar{t}>0$,

$$
y_{n}(t) \rightarrow y_{0}(t), \quad y_{n}^{\prime}(t) \rightarrow y_{0}^{\prime}(t)
$$

as $n \rightarrow \infty$, uniformly on $[0, \bar{t}]$.

By standard theory, $u_{n}(t) \rightarrow u_{0}(t)$ as $n \rightarrow \infty$ uniformly on $[0, \bar{t}]$. Therefore, $p_{n}(t) \rightarrow p_{0}(t)$ as $n \rightarrow \infty$ in $L^{1}[0, \bar{t}]$.

If for $n \geq 1$ we set $v_{n}(t)=y_{n}(t)-y_{0}(t)$ then

$$
\begin{gathered}
v_{n}^{\prime \prime}(t)+k v_{n}^{\prime}(t)+p_{n}(t) v_{n}(t)=\left(p_{0}(t)-p_{n}(t)\right) v_{0}(t), \\
v_{n}(0)=v_{n}^{\prime}(0)=0, \quad n \geq 1 .
\end{gathered}
$$

If $\bar{b}$ denotes the maximum of $b(s)$ for $0 \leq s \leq 1$, then $\left|p_{n}(t)\right| \leq \bar{b}$ for all $s \in[0,1]$. Therefore, if $\varepsilon_{n}$ denotes the $L^{1}[0, \bar{t}]$-norm of $\left(p_{n}-p_{0}\right)$ for $n \geq 1$, $c_{0}$ denotes the maximum of $\left|v_{0}(t)\right|$ on $[0, \bar{t}]$ and $M=1+k+\bar{b}$, then, from the above we have for $0 \leq t \leq \bar{t}$ and $n \geq 1$

$$
\left|v_{n}(t)\right|+\left|v_{n}^{\prime}(t)\right| \leq \varepsilon_{n} c_{0}+M \int_{0}^{t}\left(\left|v_{n}(s)\right|+\left|v_{n}^{\prime}(s)\right|\right) d s .
$$

Hence, by Gronwall's lemma

$$
\left|v_{n}(t)\right|+\left|v_{n}^{\prime}(t)\right| \leq \varepsilon_{n} c_{0} \exp M t
$$

for $0 \leq t \leq \bar{t}$ and $n \geq 1$. This shows that $v_{n}(t)=y_{n}(t)-y_{0}(t) \rightarrow 0$ and $v_{n}^{\prime}(t)=y_{n}^{\prime}(t)-y_{0}(t) \rightarrow 0$ as $n \rightarrow \infty$ uniformly on $[0, \bar{t}]$ and the proof is complete.

The first author would like to thank Raul Manasevich for pointing out some errors in a preliminary version of this paper.

\section{REFERENCES}

1. W. A. Coppel, Stability and asymptotic behavior of differential equations, Heath Math. Monographs, Heath, Boston, Mass., 1965.

2. M. G. Crandall and P. H. Rabinowitz, Bifurcation from simple eigenvalues, J. Funct. Anal. 8 (1971), 321-340.

3. Th. Gallouët and O. Kavian, Résultats d'existence et de non-existence pour certains problèmes demi-linéaires á l'infini, Ann. Fac. Sci. Toulouse Math. (5) 3 (1981), 201-246.

4. __ Resonance for jumping non-linearities, Comm. Partial Differential Equations 7 (1982), 325-342.

5. J. Glover, A. C. Lazer and P. J. McKenna, Existence of stability of large scale nonlinear oscillations in suspension bridges, J. Appl. Math. Phys. (ZAMP) 40 (1989), 172-200.

6. J. K. Hale, Ordinary differential equations, Wiley-Interscience, New York, 1969.

7. M. W. Hirsh and S. Smale, Differential equations, dynamical systems, and linear algebra, Academic Press, New York, 1974.

8. A. C. Lazer, Applications of a lemma on bilinear forms to a problem in nonlinear oscillations, Proc. Amer. Math. Soc. 33 (1972), 89-94.

9. A. C. Lazer and P. J. McKenna, Critical point theory and boundary value problems with nonlinearities crossing multiple eigenvalues. II, Comm. Partial Differential Equations 11 (1986), 1653-1676. 
10. __ Large scale oscillatory behaviour in loaded asymmetric systems, Ann. Inst. H. Poincaré Sect. (N.S.) 4 (1987), 243-276.

11. _ A symmetry theorem and applications to nonlinear partial differential equations, J. Differential Equations (to appear).

12. D. E. Leach, On Poincaré's perturbation theorem and a theorem of W. S. Loud, J. Differential Equations 7 (1970), 34-53.

13. W. S. Loud, Periodic solutions of $x^{\prime \prime}+c x^{\prime}+g(x)=\varepsilon f(t)$, Mem. Amer. Math. Soc. No. 31 (1959).

14. R. F. Manasevich, A non-variational version of a max-min principle, Nonlinear Anal. TMA 7 (1983), 565-570.

15. J. L. Massera, The existence of periodic solutions of systems of differential equations, Duke Math. J. 17 (1950), 457-475.

16. L. Nirenberg, Topics in nonlinear functional analysis, Courant Institute of Mathematical Sciences, 1974.

Department of Mathematics and Computer Science, University of Miami, Coral GABLES, FLORIDA 33124

Department of Mathematics, University of Connecticut, Storrs, Connecticut 06268 